\title{
Criticality experiments and benchmarks for cross section evaluation: the neptunium case
}

\author{
L.S. Leong ${ }^{1,2, a}$, L. Tassan-Got ${ }^{1,2, b}$, L. Audouin ${ }^{2,1}$, C. Paradela ${ }^{3}$, J.N. Wilson ${ }^{1,2}$, D. Tarrio ${ }^{3}$, \\ B. Berthier ${ }^{1,2}$ I. Duran ${ }^{3}$, C. Le Naour ${ }^{1,3}$ and C. Sétphan ${ }^{1,2}$ \\ ${ }^{1}$ Institut de Physique Nucléaire, CNRS \\ ${ }^{2}$ Université Paris-Sud, 91406 Orsay, France \\ ${ }^{3}$ Facultad de Fisica, Universidade de Santiago de Compostela, 15782, Spain
}

\begin{abstract}
.
The ${ }^{237} \mathrm{~Np}$ neutron-induced fission cross section has been recently measured in a large energy range (from $\mathrm{eV}$ to $\mathrm{GeV}$ ) at the $\mathrm{n}_{-} \mathrm{TOF}$ facility at CERN. When compared to previous measurement the $n_{-}$TOF fission cross section appears to be higher by $5-7 \%$ beyond the fission threshold. To check the relevance of $n \_$TOF data, we apply a criticality experiment performed at Los Alamos with a $6 \mathrm{~kg}$ sphere of ${ }^{237} \mathrm{~Np}$, surrounded by enriched uranium ${ }^{235} \mathrm{U}$ so as to approach criticality with fast neutrons. The multiplication factor $k_{\text {eff }}$ of the calculation is in better agreement with the experiment (the deviation of 750 $\mathrm{pcm}$ is reduced to $250 \mathrm{pcm}$ ) when we replace the ENDF/B-VII.0 evaluation of the ${ }^{237} \mathrm{~Np}$ fission cross section by the n_TOF data. We also explore the hypothesis of deficiencies of the inelastic cross section in ${ }^{235} \mathrm{U}$ which has been invoked by some authors to explain the deviation of $750 \mathrm{pcm}$. With compare to inelastic large distortion calculation, it is incompatible with existing measurements. Also we show that the $\bar{v}$ of ${ }^{237} \mathrm{~Np}$ can hardly be incriminated because of the high accuracy of the existing data. Fission rate ratios or averaged fission cross sections measured in several fast neutron fields seem to give contradictory results on the validation of the ${ }^{237} \mathrm{~Np}$ cross section but at least one of the benchmark experiments, where the active deposits have been well calibrated for the number of atoms, favors the $n_{-}$TOF data set. These outcomes support the hypothesis of a higher fission cross section of ${ }^{237} \mathrm{~Np}$.
\end{abstract}

\section{Introduction}

${ }^{237} \mathrm{~Np}$ is a long lived (2 My) radioactive isotope which is produced abundantly in nuclear power plants. Therefore its incineration is of interest to reduce on the long term the radiotoxicity of the final disposal. This could be in principle achieved in fast reactors, where a significant fraction of the neutron energy spectrum extends beyond the first chance fission threshold, however it requires a better knowledge of the neutron cross sections specially for fission. Its measurement has been undertaken in the past and has also been recently measured. Although some data sets are consistent within the claimed accuracy, which is usually about $3-4 \%$, some significant discrepancies show up between others, in particular

\footnotetext{
a e-mail: leong@ipno.in2p3.fr

be-mail: tassango@ipno.in2p3.fr
} 
the $n \_$TOF recent measurement which exhibits values higher by about $6 \%$ when compared to several previous measurements.

To get a clarification on this problem we adopted the method of checking the cross sections within a critical experiment. It consisted in a spherical-like assembly made of a ${ }^{237} \mathrm{~Np}$ sphere surrounded by a spherical shell of Highly Enriched Uranium (HEU) in which the neutron multiplication factor $k_{e f f}$ is measured with an accuracy of $0.36 \%$. Actually $k_{\text {eff }}$ is an integral quantity depending on many neutron reaction parameters, however many of them are accurately known, and its sensitivity to the ${ }^{237} \mathrm{~Np}$ fission cross section is high enough $\left(1.510^{-3} / \%\right)$ to get a good test. Even if it cannot be considered as a definite test, due to its integral nature, it is a good indicator of the reliability of the ${ }^{237} \mathrm{~Np}(n, f)$ cross section.

We are going to describe the critical experiment below. We give an overview of the existing data concerning the ${ }^{237} \mathrm{~Np}$ fission cross section and then how the recent data sets for the ${ }^{237} \mathrm{~Np}$ fission cross section affect $k_{\text {eff }}$ and how they compare with the experimental criticality. We also explore the hypothesis of a deficient inelastic cross section of ${ }^{235} \mathrm{U}$ and its possible impact on $k_{e f f}$, and also the effect of the $\bar{v}$ of ${ }^{237} \mathrm{~Np}$. Finally we test the ${ }^{237} \mathrm{~Np}$ fission cross section against fission rate experiments in different neutron spectra.

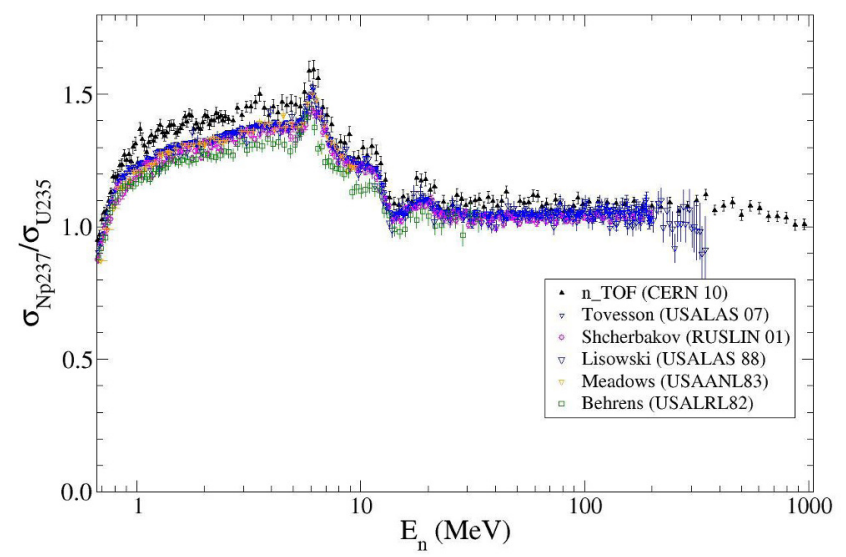

Figure 1. Comparison of $n_{-}$TOF ${ }^{237} \mathrm{~Np}$ fission cross section, relative to ${ }^{235} \mathrm{U}$

\section{Status of ${ }^{237} \mathrm{~Np}$ fission cross section}

Several experiments based on the time-of-flight technique were dedicated to the measurement of this cross section in the fast neutron region which cover a larger neutron energy range. Meadows [13] measured ${ }^{237} \mathrm{~Np}(\mathrm{n}, \mathrm{f})$ from 1 to $10 \mathrm{MeV}$ in Argonne Fast Neutron Generator (FNG) laboratory. Later Lisowski [11] measured it in Los Alamos Meson Physics Facility (LAMPF) covering the broad neutron energy range from 1 to $400 \mathrm{MeV}$. Then Shcherbakov [22] performed another measurement at the Gneiss neutron source in Gatchina. More recently, F. Tovesson [23] measured this cross section at Los Alamos Neutron Science Center, covering both subthreshold and above threshold fission from $100 \mathrm{keV}$ to $200 \mathrm{MeV}$. A comparison of the results from the above-mentioned experiments is displayed 
in Fig. 1 as a ratio to the fission cross section of ${ }^{235} \mathrm{U}$. Within the experimental uncertainties, all these data are consistent with each other, except the Behrens measurement [3]

Recently, an experiment has been performed at the n_TOF neutron facility at CERN with a neutron spectrum covering an energy range $0.7 \mathrm{eV}-1 \mathrm{GeV}[19,20]$. While being in good agreement with previous measurements and evaluated data files below and around the threshold region, the $n$ TOF's data exhibit an excess of $6-7 \%$ above $1 \mathrm{MeV}$.

This seemingly singular behavior might shed some doubt on the level of accuracy of the n_TOF results. However, one should be aware that several of the previous results are not independent because they have been renormalized to older ones. For example in Tovesson's measurement the cross section has been normalized to the ENDF/B-VI.8 nuclear data at $14.8 \mathrm{MeV}$ because the amount of target material was not known with the desired precision. This evaluated data file is based on Lisowski's measurement which was normalized to Meadows' data over the 1 to $10 \mathrm{MeV}$ energy range for similar reasons concerning the targets. Therefore the consistency of several experimental data sets, and of the derived evaluated libraries, is in the first place a consequence of these normalizations.

The mismatch between the fission cross sections should be investigated properly. We made one step in this direction by testing them in the simulation of a critical assembly containing a significant quantity of ${ }^{237} \mathrm{~Np}$, and comparing the computed $k_{e f f}$ to its value obtained experimentally.

\section{The critical ${ }^{237} \mathrm{~Np}$ benchmark}

\subsection{The composite critical experiment}

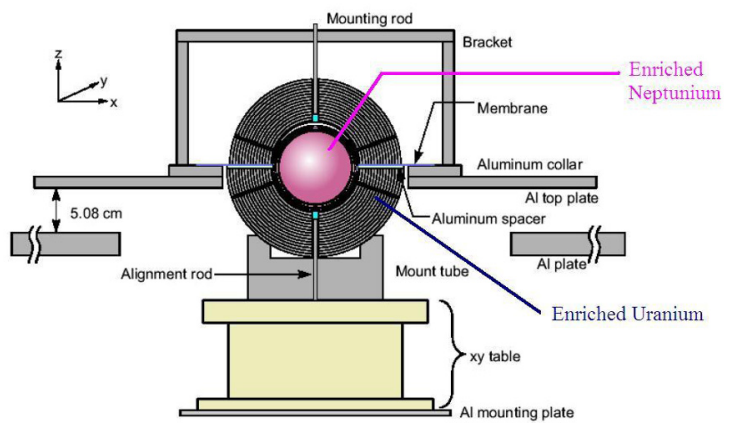

Figure 2. Detailed scheme of the composite $\left({ }^{237} \mathrm{~Np}\right.$ and HEU) assembly in compact configuration

There is a benchmark experiment with a composite assembly associating neptunium and HEU made at Los Alamos. The aim was to define better the critical mass of ${ }^{237} \mathrm{~Np}$ [12, 21]. This experiment is based on a ${ }^{237} \mathrm{~Np}$ sphere of $6 \mathrm{~kg}$ surrounded by nested hemispherical shells of HEU which contains $85 \%$ of the fissile mass, as sketched in Fig. 2.

${ }^{237} \mathrm{~Np}$ is less fissile than ${ }^{235} \mathrm{U}$ due to its threshold, but fast neutrons of energy higher than $0.6 \mathrm{MeV}$ can trigger its fission. Therefore the $k_{\text {eff }}$ is significantly sensitive to the ${ }^{237} \mathrm{~Np}$ cross sections, especially to the fission one.

The configuration where the criticality is reached $\left(k_{e f f}=1\right)$ is determined by extrapolating linearly the inverse of the number of detected neutrons [21]. When all components are in contact, criticality was determined to be $k_{e f f}=1.0026 \pm 0.0036$, that is 360 pcm uncertainty. 


\subsection{The critical neptunium benchmark}

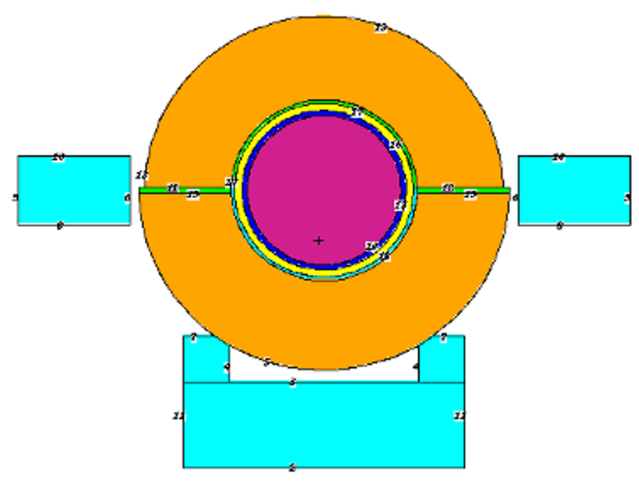

Figure 3. Neptunium's benchmark geometry. The right-hand side rectangle is an expanded view of a part of the spherical-like assembly.

To simulate easily this experiment as a benchmark, Mosteller and co-workers [16, 17], applied some simplifications to the geometry by homogenizing some parts with the actual small gaps and by approximating outer structural materials. As the benchmark is very close to the real experiment, the variation of $k_{e f f}$ is small and can be reliably calculated. The resulting value is $k_{e f f}=1.0019 \pm 0.0036$. This benchmark geometry is represented in Fig. 3 and we used it in all the simulations.

We computed the benchmark with MCNP5 (Monte Carlo code for neutron transport) [25] driven by MURE (MCNP Utility for Reactor Evolution) [14]. The default cross sections were those from the evaluated library ENDF/B-VII.0 [4]. In such conditions the criticality is caculated to be $k_{\text {eff }}=0.99418 \pm 0.00006$, very close to the value computed by the Los Alamos group [15]. The deviation from the benchmark value is $-770 \mathrm{pcm}$, more than twice the experimental uncertainty. This arouses suspicions about the ${ }^{237} \mathrm{~Np}$ cross sections.

It is worth pointing out that the ${ }^{237} \mathrm{~Np}$ fission cross section in ENDF/B-VII.0 is essentially based on Tovesson's measurement. As the n_TOF cross section is higher by about $6 \%$ above $1 \mathrm{MeV}$ it could help to increase the $k_{e f f}$ and bring it closer to the benchmark value. To check this hypothesis we replaced the ENDF/B-VII.0 ${ }^{237} \mathrm{~Np}$ fission cross section. All the other cross sections were left unchanged, therefore the total reaction cross section was scaled to accommodate the variation of the fission cross section. After this substitution, we obtained $k_{e f f}=1.00435 \pm 0.00006$. Although it exceeds slightly the benchmark value, the deviation is significantly reduced to $250 \mathrm{pcm}, 0.7$ times the experimental uncertainty. As $k_{e f f}$ is sensitive to other parameters, this finding cannot be taken as definitely conclusive on the better fission cross section set. Yet it indicates that the hypothesis of a ${ }^{237} \mathrm{~Np}$ fission cross section higher than expected before should be considered seriously.

\section{Role of inelastic cross section of ${ }^{235} \mathrm{U}$}

The disagreement between the experimental criticality and its simulated value when using the ENDF/B-VII.0 library was already noted by some authors who ascribed it to a possible deficiency 
of the inelastic cross section in ${ }^{235} \mathrm{U}$ which was also suspected from the under-prediction of fission rate ratios ${ }^{237} \mathrm{~Np} /{ }^{235} \mathrm{U}$ at the center of several critical experiments [4].

Therefore, We are going to study how this cross section affects the $k_{\text {eff }}$ in the benchmark configuration and how the variation required to match the experimental value compares with measurements of the inelastic cross section.

\subsection{Impact of the ${ }^{235} \mathrm{U}$ inelastic cross section}

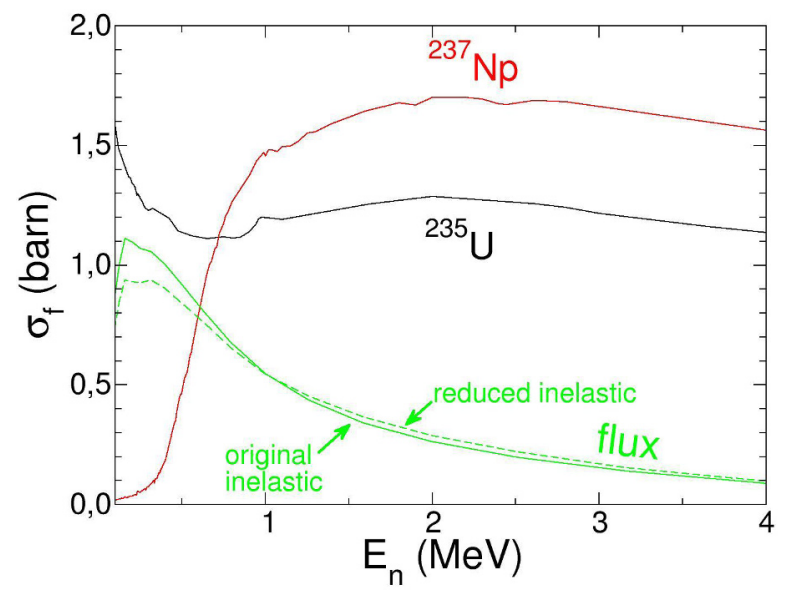

Figure 4. Effect of a distortion of the inelastic cross section on the neutron flux and fission cross sections of ${ }^{237} \mathrm{~Np}$ and ${ }^{235} \mathrm{U}$

The $\left(\mathrm{n}, \mathrm{n}^{\prime}\right)$ reaction is treated as a set of individual cross sections. Changes in the set of inelastic cross sections alter the neutron spectrum. For example, if the cross section for the highest levels is decreased while it is increased for the lowest ones, outgoing neutrons are expected to have a higher average energy. This is illustrated in Fig. 4 where the bell-shaped curves represent the energy spectrum of the neutron flux in the ${ }^{237} \mathrm{~Np}$ sphere. The dashed curve, labeled reduced inelastic, results from a modification of the inelastic cross sections as described above. As expected, the flux is shifted toward higher energies. The fission cross section of ${ }^{235} \mathrm{U}$ is overall flat, so its fission rate is weakly sensitive to shift in the flux. Conversely, ${ }^{237} \mathrm{~Np}$ has a fission threshold, hence a harder neutron flux will increase the fission rate (dashed curve).

In order to evaluate the modifications on the ${ }^{235} \mathrm{U}$ inelastic cross sections and if it may reconcile the criticality of benchmark, we have performed calculations using various sets of ${ }^{235} \mathrm{U}$ inelastic cross sections and the total cross section was affected by the variation of the inelastic cross section.

In MCNP, forty levels are described (MT=51 to 90, starting with the first excited level) ; they sit on top of the continuum (MT=91, a large number of excited levels are included, mainly located at high excitation energy). Therefore we adopted a random procedure to vary these cross sections. Not all sets of the modifications are acceptable because the inelastic cross section of ${ }^{235} \mathrm{U}$ also affects its critical mass which is well known. In other words the modification of the inelastic cross section should not change the computed criticality of a HEU sphere.

Therefore, we checked this feature by running a MCNP calculation with each of the proposed cross section sets over the GODIVA benchmark (HEU-MET-FAST-001) describing a bare HEU sphere 
reaching criticality [10]. Among 8000 sets generated, only 551 were validated using the GODIVA benchmark, and were then used for the computation of the ${ }^{237} \mathrm{~Np}$ benchmark.

Fig. 5 displays the $k_{\text {eff }}$ obtained in these 551 computations. As expected it peaks at the unperturbated value for the ${ }^{237} \mathrm{~Np}$ benchmark and spreads rather narrowly around its average. Nevertheless, a few values reach the experimental range. This means that some specific modifications of the inelastic cross section comply with the conservation of the ${ }^{235} \mathrm{U}$ critical mass and have a good agreement with the measured criticality for the ${ }^{237} \mathrm{~Np}$ benchmark. These sets reveals that they are always generated by highly depressing the continuum contribution ( $\mathrm{MT}=91$ ). Therefore the required shift of the energy spectrum can only be obtained by reducing strongly its contribution in favor of lower lying levels. This is shown in fig. 5 where the distributions of calculated $k_{\text {eff }}$ are labelled with the reduction of the continuum part. The statistics drop steeply when the level of reduction is enhanced because it becomes more difficult to find cross section configurations conserving the criticality of ${ }^{235} \mathrm{U}$. In the following, we intend to check the consistency of such an outcome with existing measurements.

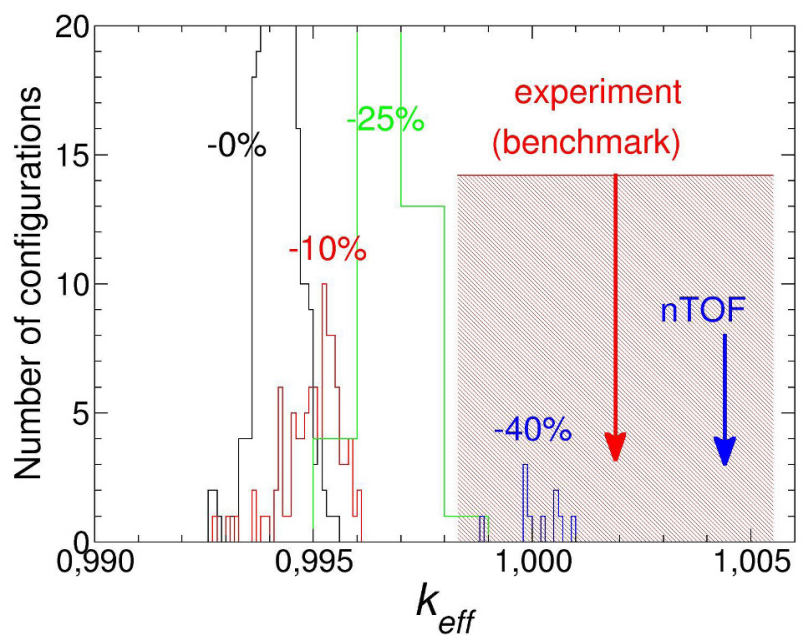

Figure 5. Histograms of $k_{e f f}$ for cross section configurations conserving the GODIVA criticallity and for different level of reduction of the continuum part.

\subsection{Comparison to previous inelastic measurements}

Knitter used a Van de Graaff accelerator to produce mono-energetic neutron beams of 1.5, 1.9 and 2.3 $\mathrm{MeV}$ [9] in order to measure the inelastic scattering cross section of ${ }^{235} \mathrm{U}$. The kinetic energy loss of neutrons was integrated over $200 \mathrm{keV}$-wide bands from $0.4 \mathrm{MeV}$ to the incident energy minus $0.5 \mathrm{MeV}$.

For the simulation fig. 6 shows the energy spectrum of the outgoing neutron for a $2.3 \mathrm{MeV}$ incoming neutron, as extracted from ENDF/B-VII.0. The peaks, widened by the recoil kinematics, are produced by populating discrete excited levels of the remaining ${ }^{235} \mathrm{U}$ (MT=51 to 90), whereas the smooth spectrum represents the continuous part ( $\mathrm{MT}=91$ ) ending at an energy corresponding to the minimum excitation energy of $\approx 0.5 \mathrm{MeV}$.

The spectra were then integrated spectra over bands of $200 \mathrm{keV}$ to allow a direct comparison with Knitter's data, as shown in fig. 7 The ENDF/B-VII.0 evaluation is close to Knitter's measurement, 


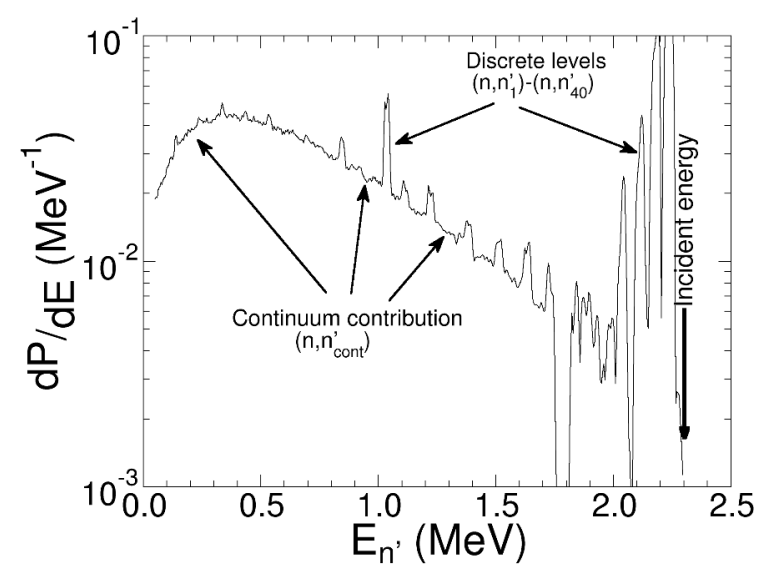

Figure 6. Energy spectrum of the outgoing inelastically scattered neutron as simulated for ${ }^{235} \mathrm{U}$ using ENDF/BVII.0 inelastic cross sections (MT=51 to 91), for an incident energy of $2.3 \mathrm{MeV}$ (reaction probability).

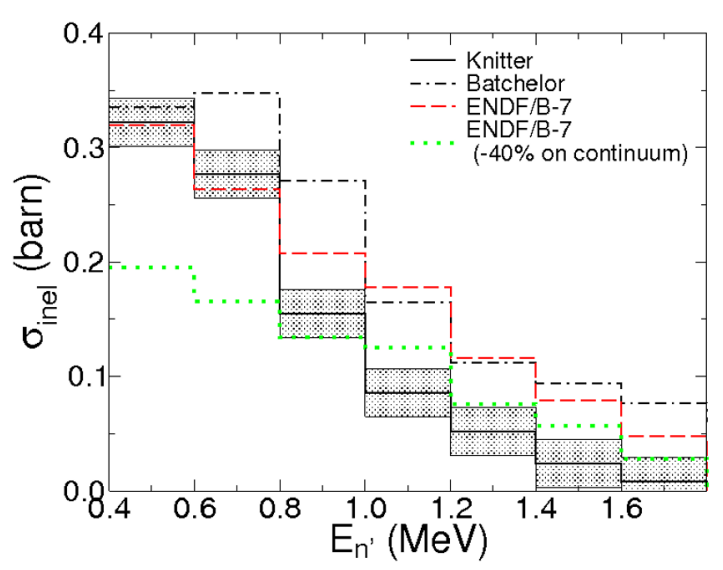

Figure 7. Comparison of inelastic neutron scattering cross sections of ${ }^{235} \mathrm{U}$ (barn) for energy bands of $200 \mathrm{keV}$ for the outgoing neutron and for an incoming energy of $2.3 \mathrm{MeV}$. Solid line: Knitterś values with the error bars represented by the hatched rectangles. Dashed line: ENDF/B-VII.0.

although a systematic overestimation shows up beyond $0.8 \mathrm{MeV}$. Batchelor's data [2] ares also plotted and the agreement with ENDF/B-VII.0 is generally even better than in the case of Knitter's data, although a significant discrepancy appears between 0.6 and $1 \mathrm{MeV}$.

In fig. 7 is also plotted a typical spectrum generated by a cross section configuration with $40 \%$ reduction of the continuum contribution. As expected, the low energy part of the spectrum is depleted and compensated by a strengthening of the part leading to low lying states, hence to high scattered neutrons energy : this will lead to the desired increase of $k_{\text {eff }}$ in the ${ }^{237} \mathrm{~Np}$ benchmark as some of the low energy neutrons are put beyond the fission threshold. Although such a cross section set seems to be in better agreement with Knitter's measurement above $0.8 \mathrm{MeV}$, it fails strongly below this energy. 
The main difficulties of such measurements is the subtraction of fission neutrons from the neutron spectra. In fact, a lower energy of the scattered neutrons is less polluted by the fission contribution, therefore, we consider the low-energy part results more reliable than the high energy part, an assumption which is corroborated by the consistency of the two measurements, which gets better as one looks at lower scattered neutrons energy. The $-40 \%$ configuration is strongly discrepant with experimental data in the more reliable region. We conclude that it doesn't reconcile the measurement of the $k_{\text {eff }}$ of the ${ }^{237} \mathrm{~Np}$ benchmark with simulation, because it requires a $40 \%$ reduction of the continuum part of ${ }^{235} \mathrm{U}$ inelatic cross section, which is hardly consistent with the data.

\section{Influence of ${ }^{237} \mathrm{~Np} \bar{v}$}

As the criticality experiment is in fact an integral measurement which $k_{\text {eff }}$ is also sensitive to other parameters beside cross sections. The average number of emitted neutrons per fission $\bar{v}$ is also an important parameter for the criticality. As the ${ }^{235} \mathrm{U} \bar{v}$ is well constrained by HEU critical experiments we only apply variations to the ${ }^{237} \mathrm{~Np} \bar{v}$.

The simulation shows that a $3 \%$ increase on ${ }^{237} \mathrm{~Np} \bar{v}$ (prompt+delayed) produces a $k_{\text {eff }}$ shift of $766 \mathrm{pcm}$ even though only $13 \%$ of fissions occur in the neptunium sphere. Therefore an increase of about $3 \%$ of $\bar{v}$ is needed to make $k_{\text {eff }}$ reach the experimental value.

Fig. 8 shows the $\bar{v}$ energy dependence for several experimental sets. For sake of clarity the error bars are not reported in the figure but they lie mostly between 0.5 and $1.5 \%$. Most of the experimental values are lower than the evaluated ones, except Veeser's data set around $7 \mathrm{MeV}$, which is well above the mean energy of neutrons in the critical device. This comparison doesn't support the plausibility of an underestimation of the total $\bar{v}$ that would explain the mismatch of the benchmkark criticality.

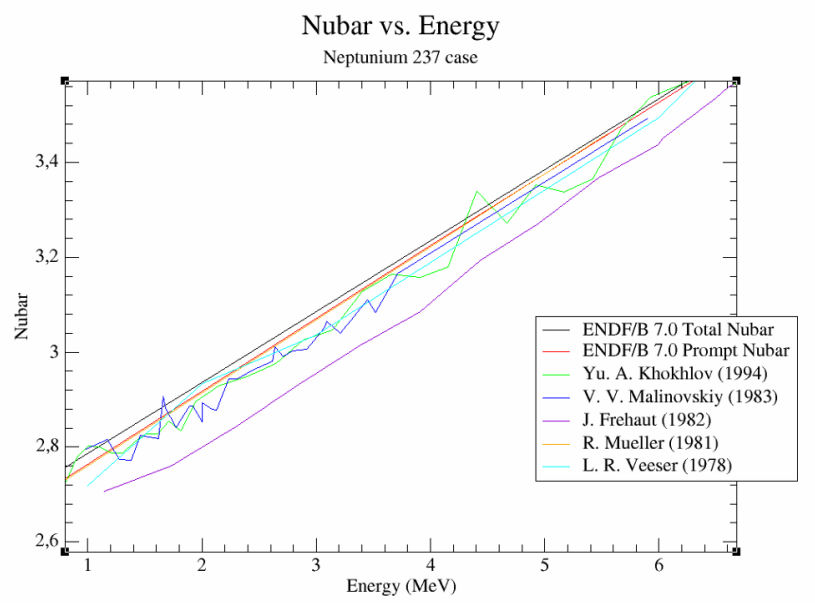

Figure 8. For fission of ${ }^{237} \mathrm{~Np}$ energy dependence of $\bar{v}$ as measured and extracted from ENDF7.0

\section{Fission rate experiments}

Fission rate measurements consist of recording the number of fissions, with fission detectors, for example ionization chambers, when a sample is subject to a neutron field of known spectrum. The 
rate is directly related to the fission cross section. One can define the averaged cross section as:

$$
<\sigma>=\frac{\int W(E) \sigma(E) d E}{\int W(E) d E}
$$

where $W(E)$ is the neutron energy spectrum. And the fission rate ratio, or spectral index, as the ratios of averaged cross sections:

$$
R_{f}=\frac{\left\langle\sigma>_{N p 237}\right.}{\left\langle\sigma>_{U 235}\right.}
$$

ENDF/B-VII.0 and ENDF/B-VII.1 [5] libraries have been tested with different fission rate experiments [7].

The first situation we consider is the case of fission rates measured at the center of a GODIVA assembly. It is an enriched ${ }^{235} \mathrm{U}$ sphere [10] and the ratio of fission rates is obtained with ${ }^{237} \mathrm{~Np}$ and ${ }^{235} \mathrm{U}$ samples by dividing the former rate by the latter. Table 1 (first line) shows the comparison between this measured ratio, its simulated value when using the ENDF/B-VII.0 library, and the computed value when the n_TOF ${ }^{237} \mathrm{~Np}$ fission cross section is used. The ENDF/B-VII.0 underestimates the result by $2.4 \%$ whereas the $\mathrm{n}_{-} \mathrm{TOF}$ data is higher by $3.5 \%$.

Table 1. Comparison of experimental and simulated fission rates and averaged fission cross section

\begin{tabular}{|c|c|c|c|c|}
\hline Observable & Set-up & Exp. & Calc. (ENDF/B-VII.0) & Calc. (n_TOF) \\
\hline$R_{f}$ & GODIVA(HMF001-002) & $0.8516(12)$ & 0.83 & 0.88 \\
& MASURCA(COSMO) & 0.285 & 0.284 & 0.299 \\
\hline$<\sigma>$ & DOSIMETRY & $1.361(22)$ & 1.357 & 1.431 \\
\hline
\end{tabular}

We also looked at the COSMO benchmark which is a well defined critical configuration of MASURCA $(180 \mathrm{~cm} \times 180 \mathrm{~cm} \times 164.2 \mathrm{~cm})$ [18] made of 3 parts, which compositions are homogenised: MOX core, reflector and shielding. The fission rates of ${ }^{237} \mathrm{~Np}$ and ${ }^{235} \mathrm{U}$ have been measured at several points but we refer here to the central measurement where the flux has a maximum. Again table 1 shows the experimental ratio ${ }^{237} \mathrm{~Np} /{ }^{235} \mathrm{U}$ and the results of the computations. From this comparison it turns out that the n_TOF cross section is too high by $5 \%$ whereas the ENDF/B-VII.0 is very close to the experimental value.

The last situation we will discuss is the case of a neutron field generated by the spontaneous fission of a ${ }^{252} \mathrm{Cf}$ source. The fission rates from ${ }^{237} \mathrm{~Np}$ and ${ }^{235} \mathrm{U}$ deposits in ionization chambers are recorded when they are placed at different distances from a stainless steel spherical shell containing a ${ }^{252} \mathrm{Cf}$ source. Instead of carrying a full simulation of the experiment we just performed a simple calculation of the fission averaged fission cross section by assuming a pure Watt spectrum coming from the spontaneous fission of ${ }^{252} \mathrm{Cf}$, with $a=0.84746 \mathrm{MeV}^{-1}$ and $b=1.03419 \mathrm{MeV}^{-1}$ [24], which is also the parametrization adopted in ENDF/B-VII. We applied a correction for the tiny moderation of the neutrons. This correction has been estimated from the difference of the unperturbated and moderated fluxes as computed in [8], and it amounts to $1.2 \%$.

This comparison of fission rates seems to be puzzling because it leads to contradictory conclusions according to the different experiments. However in the case of COSMO the sample amounts have been determined through neutron cross sections, and this cannot be retained as a test of the cross section which precisely has been used for the calibration. This stresses the importance of calibrations of the samples independent of cross sections, for example through alpha counting. In the GODIVA case we don't have any information on the calibration procedure of the samples which have been used and the description of the experimental details and the method used to calibrate the fission rate is missing. This also applies to to the measurement used for dosimetry. 


\section{Conclusion}

We used the ${ }^{237} \mathrm{~Np}$ critical benchmark to test the validity of the ${ }^{237} \mathrm{~Np}$ fission cross section measured at n_TOF, which appeared to be larger than previous measurements. The $k_{\text {eff }}$ predicted using the n_TOF cross section, although slightly exceeding the experimental value, is much closer to the benchmark value and falls inside the uncertainty range whereas this was not the case for older ${ }^{237} \mathrm{~Np}$ fission cross sections. As some authors invoked a possible deficiency of the $\left(n, n^{\prime}\right)$ cross section in ${ }^{235} \mathrm{U}$ to explain the mismatch we investigated this hypothesis. It turns out that the experimental range can be reached only by depressing the continuum contribution of the inelastic cross section by at least $40 \%$. Such a modification is hardly consistent with existing measurements of the ${ }^{235} \mathrm{U}$ inelastic cross section. Beside, we showed that the discrepency can't be ascribed to the ${ }^{237} \mathrm{~Np} \bar{v}$. Although other effects and uncertainties can affect the criticality, our prediction of the criticality of the ${ }^{237} \mathrm{~Np}$ benchmark may indicate that the ${ }^{237} \mathrm{~Np}$ fission cross section is higher than expected from previous measurements. When testing the ${ }^{237} \mathrm{~Np}$ fission cross section with fission rate experiments the outcomes are not univoque, but we showed that the calibration procedure of the detectors is of crucial importance and its description is often lacking. Although this can't be considered as definitely conclusive, it indicates that a global increase of the ${ }^{237} \mathrm{~Np}$ fission cross section by $4-5 \%$, against the data libraries and more peculiarly ENDF/B-VII.0, could not be discarded.

\section{References}

[1] Adamov V.M. et al., Gaithersburg, March 28-31, 1977

[2] Batchelor R. and Wyld K., EXFOR 20036.005

[3] Behrens J.W. et al.,Nuclear Science and Engineering 85, 271-279, (1983).

[4] Chadwick M.B. et al.,Nucl. Data Sheets 107, 2931-3060 (2006)

[5] Chadwick M.B. et al.,Nucl. Data Sheets 112, 2887-2996 (2011)

[6] Grundle J.A. et al.,Nuclear Technology 25, 237-257, (1975).

[7] Kahler, A.C. et al.,Nuclear Data Sheets 112, 2997-3036 (2011)

[8] Kim S.S. et al.,NEA/NSC/DOC/(95)03/II.

[9] Knitter, H.-H. et al.,Z. Phys. 257, 108-123 (1972)

[10] LaBauve R.J. et al.,NEA/NSC/DOC/(95)03/II.

[11] Lisowski P.W. et al.,Japan, 30 May-3 Jun. 1988, p. 97

[12] Loaiza D. et al.,NEA/NSC/DOC/(95)03/VII

[13] Meadows J.W. et al.,Nuclear Science and Engineering 85, 271-279 (1983).

[14] Meplan, O. et al.,http://www.nea.fr/tools/abstract/detail/nea-1845, April 2009.

[15] Mihaila B. et al.,LA-UR-04-1959 (2004).

[16] Mosteller R.D. et al.,LA-UR-04-0525, (2004).

[17] Mosteller R.D. et al., Mosteller R.D. et al.,

[18] NEA/NSC/DOC(2005)23

[19] Paradela C. et al.,Phys. Rev. C 82, 034601,(2010).

[20] Paradela C. et al., Physical Society, Vol. 59, No. 2, 1908 (2011)

[21] Sanchez R. et al.,Nuclear Science and Engineering 158, 1-14 (2008).

[22] Shcherbakov O. et al.,J. Nuclear Science Technology 2, 230-233 (2002).

[23] Tovesson F. and Hill T.S.,Phys. Rev. C 75, 034610 (2007).

[24] Verbeke J.M., Hagmann C., Wright D.,UCRL-AR-228518 (2010)

[25] Los Alamos National Laboratory report LA-UR-03-1987 (April 2003) 